\title{
The Impact of Low Muscle Mass in Patients Undergoing Emergency Surgery for Colonic Perforation - A Single-center Experience
}

\author{
HAJIME IMAMURA ${ }^{1}$, MASASHI HARAGUCHI ${ }^{1}$, SHIGEKI MINAMI ${ }^{1}$, YURIKO ISAGAWA ${ }^{1}$, \\ MICHI MORITA ${ }^{1}$, MASATAKA HIRABARU ${ }^{1}$, DAISUKE KAWAHARA ${ }^{1}$, \\ HIROTAKA TOKAI $^{1}$, KAZUMASA NODA $^{1}$, KEIJI INOUE ${ }^{1}$ and SUSUMU EGUCHI $^{2}$ \\ ${ }^{1}$ Department of Surgery, Nagasaki Harbor Medical Center, Nagasaki, Japan; \\ ${ }^{2}$ Department of Surgery, Nagasaki University Graduate School of Biomedical Sciences, Nagasaki, Japan
}

\begin{abstract}
Aim: The aim was to analyze the correlation between psoas muscle mass and mortality, as well as postoperative complications in patients treated for colonic perforation. Patients and Methods: A total of 46 patients met the study criteria. Patients were classified into an elderly (age, $\geq 75$ years, $n=24$ ) and a younger group (age, $<75$ years, n=22). Background factors, postoperative data (including duration of hospital stay and discharge) were collected. The cross-sectional area of the psoas muscle area (PMA) was measured on the same day of operation. Results: The agellength of stay and PMA were significantly correlated in the younger group $(p=0.0015,0.023$, respectively). Fifteen and six patients were discharged to return home, and 8 and 16 patients were transferred to another hospital, in the younger and elderly groups, respectively ( $p=0.02$ ). Discharge was not correlated with the PMA in either group. Conclusion: The total psoas muscle mass would be useful as a quick and convenient measure of sarcopenia in younger patients, but not elderly patients.
\end{abstract}

Sarcopenia is a syndrome characterized by a progressive and generalized loss of skeletal muscle mass and strength and is associated with an increased risk of adverse outcomes, such as physical disability, a poor quality of life,

This article is freely accessible online.

Correspondence to: Susumu Eguchi, MD, Ph.D., Department of Surgery, Nagasaki University Graduate School of Biomedical Sciences, 1-7-1 Sakamoto, Nagasaki 852-8501, Japan. Tel: +81 958197316, Fax: +81958197319, e-mail: sueguchi@nagasakiu.ac.jp

Key Words: Low muscle mass, sarcopenia, emergency surgery, colonic perforation, elderly, abdominal surgery. and death $(1,2)$. Recent studies have reported that sarcopenia is associated with worse outcomes in patients undergoing surgical resection of primary and metastatic colorectal cancer, pancreatic cancer, and liver transplantation (3-7). The presence of sarcopenia also predicts greater mortality in patients with liver cirrhosis, melanoma, and those awaiting liver transplantation (8-10). The diagnosis of sarcopenia requires documentation of low skeletal muscle plus either low muscle strength or low physical performance (11). However, it is difficult to evaluate muscle function (strength or performance) in the clinical setting, especially in acute-care surgery. The measurement of sarcopenia in the acute-care surgical population should be quick and convenient. Thus, the psoas muscle mass is often used because the evaluation of the psoas muscle is readily available with existing preoperative imaging, there was precedent in the literature on sarcopenia, and because change in the core muscle has been considered an indication related to global health and chronic illness $(3,12)$.

Colonic perforation has also been associated with high rates of morbidity and mortality in patients undergoing acute-care surgery. The establishment of an objective and patient-specific predictor of adverse outcomes would be useful for preoperative discussion, decision-making, and postoperative planning for such patients. Furthermore, with the increasing aging population, the number of elderly patients undergoing emergency surgery is increasing. The loss of muscle mass and function with aging is a practical measure of frailty and has been identified as a predictor of outcomes in elderly patients $(13,14)$.

From this background, we analyzed the correlation between the psoas muscle mass and mortality as well as the development of postoperative complications in younger and elderly patients undergoing emergency surgery for colonic perforation. 
Table I. Patient characteristics.

\begin{tabular}{lccc}
\hline Characteristic & Younger group (aged $\leq 74$ years) & Elderly group (aged $\geq 75$ years) & $p$-Value \\
\hline Male:female, $\mathrm{n}$ & $13: 9$ & $8: 16$ & 0.137 \\
Age, years & $64.5(36-74)$ & $81.5(75-95)$ & $<0.0001$ \\
$\mathrm{BMI}, \mathrm{kg} / \mathrm{m}^{2}$ & $22.6(18.7-36.1)$ & $19.9(16.1-24)$ & 0.0029 \\
$\mathrm{WBC}, \mathrm{n} / \mu \mathrm{l}$ & $11,250(2,000-20,600)$ & $6,700(1,000-21,900)$ & 0.024 \\
$\mathrm{CRP}, \mathrm{mg} / \mathrm{dl}$ & $10.2(0.02-34.5)$ & $4.9(0.02-39.4)$ & 0.30 \\
Albumin, g/dl & $3.6(2.5-5), \mathrm{n}=15$ & $3.1(1.9-4.1), \mathrm{n}=19$ & 0.02 \\
BUN, g/dl & $16.7(5.7-51.3)$ & $19.05(11.3-79.4)$ & 0.14 \\
Creatinine, g/dl & $0.76(0.46-1.69)$ & $0.75(0.42-8.05)$ & 0.38 \\
Total psoas area, $\mathrm{mm}^{2}$ & $1,622(681-3,105)$ & $961.5(606-1,778)$ & $<0.0001$ \\
\hline
\end{tabular}

BMI: Body mass index, WBC: White blood cell, CRP: C-reactive protein, BUN: Blood urea nitrogen. Data are the mean (range).

\section{Patients and Methods}

This study was approved by the Ethics Board of our Institute (\#1803002). All data were collected in a retrospective manner. The study included onsecutive patients who underwent emergency surgery for colonic perforation at our Institute from December 2013 to December 2017. Patients with perforated appendicitis or postoperative leakage were excluded. Patients who were $\geq 75$ years of age were classified into the elderly group, while those 74 years of age and under were classified into the younger group. The patients' baseline characteristics, preoperative data, intraoperative factors, and postoperative outcomes were also collected.

To evaluate sarcopenia, the total psoas area in the superior margin of the L4 vertebra was measured on day of surgery. The total psoas area was measured by determining the total cross-sectional area of the right and left psoas muscles. The level of the fourth lumbar vertebra was chosen based on the literature on sarcopenia $(3,12,14)$. The level of the L4 vertebra was identified, and the total psoas area was measured at the superior margin of the L4 vertebra by manually tracing the outline of the muscle. The total psoas area was measured in $\mathrm{mm}^{2}$.

Statistical analyses. Chi-squared and Fisher's exact tests were used to compare categorical variables and Student's $t$-test or MannWhitney test were used to compare continuous variables, as appropriate. Pearson's correlation coefficient was used to analyze the associations between the psoas muscle mass and age, length of stay and discharge. $p$-Values of less than 0.05 were considered to indicate statistical significance. GraphPad PRISM version 5 software (GraphPad Software, San Diego, CA, USA) was used to perform the statistical analyses and create figures.

\section{Results}

A total of 46 patients met our criteria. The patient characteristics are shown in Table I. The study population included 21 male patients and 25 female patients. A total of 22 and 24 patients were classified into younger and elderly groups, respectively. The average age of the patients in the younger and elderly groups was 64.5 (36-74) years and 81.5 (75-95) years, respectively. Body mass index, white blood cell count and albumin values of the elderly group were significantly lower than those of the younger group. The number of albumin samples was insufficient in both groups (young $\mathrm{n}=15$, elderly $\mathrm{n}=19$ ). The total psoas area of the younger group was significantly greater (by almost $70 \%$ ) than that of the elderly group $(p<0.0001)$.

The data on the intraoperative factors, including the diagnosis and types of procedure, are shown in Table II. The main causes of colonic perforation in both groups were malignant neoplasm, perforated diverticulitis and perforated colon. Partial colectomy with end colostomy was the most common procedure in this study. There were $16(72.7 \%)$ cases of panperitonitis in the younger group and $20(76.9 \%)$ in the elderly. The operative time of the elderly group was significantly shorter.

Postoperative complications and outcomes, including mortality, duration of hospital stay, and discharge, are shown in Table III. Various types of postoperative complications were experienced. With regard to mortality, one patient in the younger group died; there was no mortality in elderly group. The duration of hospital stay of the two groups did not differ to a statistically significant extent.

With regard to discharge, younger patients tended to return home, while elderly patients tended to be transferred to another hospital for further inpatient care and recovery $(p=0.02)$. A significant negative correlation between age and the total psoas area was detected in the younger group $(\mathrm{R}=0.40, p=0.0015)$, but not in the elderly $(\mathrm{R}=0.12, p=0.08)$ (Figure $1 \mathrm{~A})$, while a significant negative correlation was observed between the length of stay and the psoas area in the younger group $(\mathrm{R}=0.23$, $p=0.023$ ) (Figure 1B). In elderly group, there were no significant differences between the length of stay and the psoas area $(\mathrm{R}=0.026, p=0.45)$ (Figure $1 \mathrm{~B})$. There were no significant differences in total psoas area according to discharge in the elderly ( $p=0.20)$ nor younger $(p=0.28)$ groups (Figure 2$)$.

\section{Discussion}

This study demonstrated that a low total psoas muscle mass was associated with an increased postoperative length of stay after emergency surgery for colonic perforation in younger 
Table II. Intraoperative findings.

\begin{tabular}{lcc}
\hline Characteristic & Younger group (aged $\leq 74$ years) & Elderly group (aged $\geq 75$ years) \\
\hline Diagnosis, $\mathrm{n}$ & & 8 \\
Malignant neoplasm & 6 & 6 \\
Perforated diverticulitis & 5 & 9 \\
Perforated colon & 2 & 0 \\
Penetrated diverticulitis & 0 & 1 \\
Diverticulitis & & \\
Procedure, $\mathrm{n}$ & 2 & 3 \\
Partial colectomy & 10 & 15 \\
Partial colectomy with end colostomy & 0 & 1 \\
Partial colectomy with ileostomy & 6 & 2 \\
Colostomy & 0 & 1 \\
Ileostomy & 1 & 0 \\
Laparoscopic partial colectomy with end colostomy & 1 & 1 \\
Laparoscopic partial colectomy with ileostomy & 2 & 1 \\
Other & $16(72.7)$ & $20(76.9)$ \\
Panperitonitis, $n$ (\%) & $217.5(114-401)$ & $165(86-276)$ \\
Mean operative time (range), min* & $277.5(5-1,200)$ & $95(5-1,152)$ \\
Mean blood loss (range), ml ${ }^{\#}$ & & \\
\hline
\end{tabular}

Significantly different at $* p=0.004$ and ${ }^{\#} p=0.19$.

Table III. Postoperative complications and outcomes.

\begin{tabular}{lcc}
\hline Demographics & Younger group (aged $\leq 74$ years) & Elderly group (aged $\geq 75$ years) \\
\hline Postoperative complications, $n$ & 12 & 12 \\
DIC & 3 & 5 \\
Abscess & 2 & 2 \\
Aspiration pneumoniae & 1 & 2 \\
Re-perforation & 1 & 0 \\
Paralytic ileus & 2 & 0 \\
Surgical site infection & 1 & 0 \\
Stoma complication & 1 & 0 \\
Cardiac complication & 0 & 2 \\
Urinary infection & 0 & 1 \\
Sepsis & 2 & 0 \\
Unknown fever & 1 & 0 \\
Mortality & 1 & 0 \\
Mean hospital stay (range), days* & $20(9-60)$ & 19 \\
Discharged, n & & $(13-76)$ \\
Home & 15 & 8 \\
Another hospital & 6 & 16
\end{tabular}

DIC: Disseminated intravascular coagulation. Significantly different at $* p=0.72$ and ${ }^{\#} p=0.02$.

patients. On the other hand, in elderly patients, there was no correlation between the length of stay and the total psoas muscle mass, while the mortality, postoperative complications and the length of hospital stay were comparable to those of the younger population. We, therefore, consider the total psoas muscle mass to be useful as a quick and convenient measure that can be applied in the clinical setting - especially acute care surgery - to assess sarcopenia in younger patients but not elderly patients.

As a marker of sarcopenia, the psoas muscle area has the advantage of being easily measured from available clinical computed tomographic (CT) data, irrespective of the patient's condition. Cross-sectional abdominopelvic CT images have most commonly been used as a surrogate for overall skeletal muscle mass (15). As preoperative abdominopelvic CT studies are usually performed in patients 

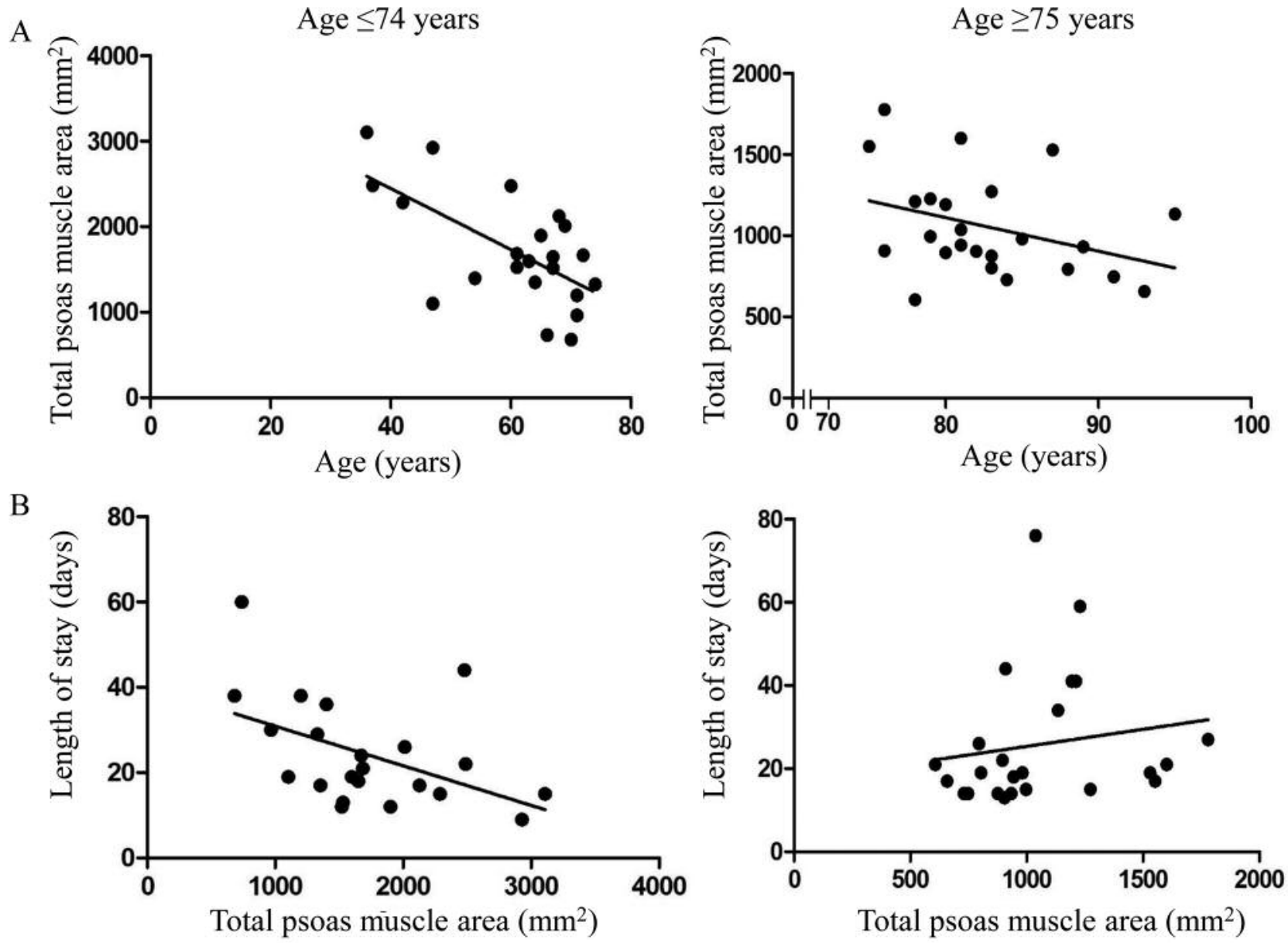

Figure 1. A: Correlation between age and the total psoas muscle area. Correlation was significant in the younger group $(R=0.40, p=0.0015)$, but not in the elderly group $(R=0.12, p=0.08)$. B: Correlation between the length of stay and psoas area. Correlation was significant in the younger group $(R=0.23, p=0.023)$, but not in the elderly group $(R=0.026, p=0.45)$.

who have clinical signs of peritonitis in the Emergency Department, these images are readily available for evaluation of the psoas muscle mass. In a previous study, sarcopenia was significantly associated with worse patient outcomes, including mortality, complications, and discharge in patients who underwent emergency operations $(13,16)$. Furthermore, Zuckerman et al. demonstrated that a low psoas muscle area was associated with an increased postoperative length of stay after cardiac surgery (17). From these points, we hypothesized that the psoas muscle mass would also be useful for evaluating postoperative outcomes, including postoperative complications, mortality, length of stay, and discharge of acute care surgery patients of all ages. However, in the present study, the psoas muscle mass was not useful for evaluating the postoperative outcomes of elderly patients. The following reasons may explain these results. Firstly, many elderly patients would have sarcopenia, regardless of their psoas muscle mass. Thus, there was no correlation between the length of hospital stay and the psoas muscle area. Secondly, the length of stay in the present study was only measured until the patient was discharged from our hospital. Many of the elderly patients were transferred to another hospital for further care and recovery. Thus, the length of stay of elderly patients was underestimated. These factors would have influenced the results for the elderly patient group.

A low psoas muscle mass would have a negative impact on the length of stay even in younger patients. The psoas muscle better reflects lower extremity muscle strength and mobility than do the lumbar and thoracic muscle groups. The psoas is a flexor of the lower extremity and a stabilizer of the lumbar vertebral column and hip joint; it therefore plays a crucial role in walking, standing from a seated position, and maintaining balance (18). Accordingly, the psoas muscle is 
Age $\leq 74$ years

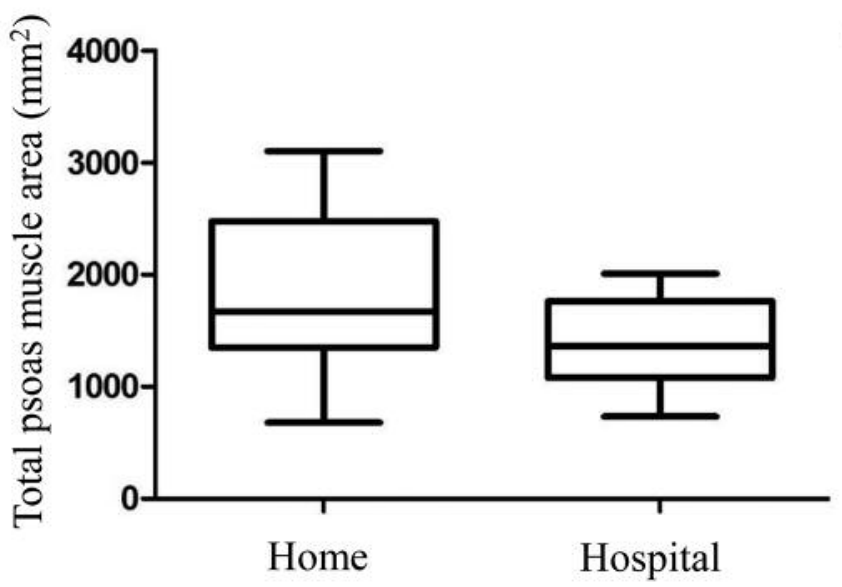

Age $\geq 75$ years

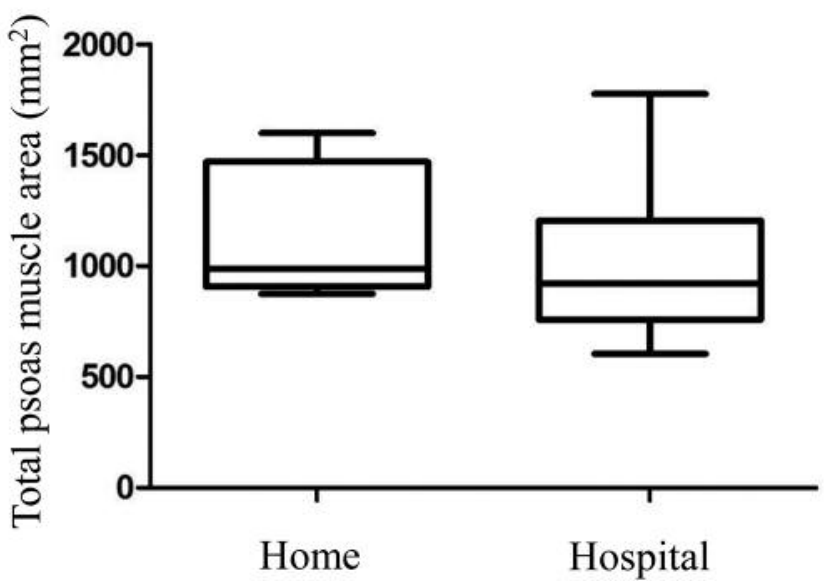

Figure 2. Total psoas area according to whether patients were discharged to their home or to another hospital. There was no significant association between the total psoas area and discharge in the elderly $(p=0.20)$ nor the younger $(p=0.28)$ group.

related to mobilization and regaining physical functions after an operation. Miller et al. reported that a larger psoas muscle area is associated with decreased odds of postoperative deficits in activities of daily living or instrumental activities of daily living after general surgery (19). Patients with a larger psoas muscle area are considered to be more capable and consequently more likely to achieve early mobilization, thereby reducing their length of hospital stay.

Sarcopenia may also reflect nutritional status, and predict infection and the ability to recover from surgery (13). A low nutritional status, also has a negative impact in relation to postoperative complications, length of stay, and discharge. At present, albumin is generally used as a measure of visceral protein and the skeletal muscle mass in clinical or nursing home settings as an evaluation of nutritional status; a low preoperative albumin level was significantly associated with poor patient outcomes (20). However, albumin is not always measured in patients undergoing emergency operations, as was shown in our study. Thus, we consider the measurement of psoas muscle mass to be a practical tool for evaluating the preoperative nutritional status. Before surgery, it is also necessary to explain the perioperative information, including the patient's condition, the treatment policy and the predicted prognosis, as well as obtaining informed consent from the patient and family, based on limited information. In this regard, the psoas muscle mass would be easy to introduce as it is a convenient and versatile measurement that can even be applied in cases involving emergency treatment. In addition, it is important to show this information to colleagues for preoperative discussion. These discussions can be used to plan tailored preventive strategies or for postoperative rehabilitation.
One limitation of the present study was the small number of patients with colonic perforation. As such, further investigations will be needed to confirm these findings.

\section{Conclusion}

Measurement of the psoas muscle area may be useful for predicting outcomes, including the length of stay in younger patients, but not for predicting the outcomes of emergency surgery in elderly patients. Assessments of the postoperative physical function are needed to further understand sarcopenia.

\section{Disclosure}

The Authors declare no conflicts of interest in association with the present study.

\section{References}

1 Goodpaster BH, Park SW, Harris TB, Kritchevsky SB, Nevitt M, Schwartz AV, Simonsick EM, Tylavsky FA, Visser $M$ and Newman AB: The loss of skeletal muscle strength, mass, and quality in older adults: The Health, Aging and Body Composition Study. J Gerontol A Biol Sci Med Sci 61(10): 1059-1064, 2006.

2 Delmonico MJ, Harris TB, Lee JS, Visser M, Nevitt M, Kritchevsky SB, Tylavsky FA, Newman AB; Health, Aging and Body Composition Study: Alternative definitions of sarcopenia, lower extremity performance, and functional impairment with aging in older men and women. J Am Geriatr Soc 55(5): 769774, 2007.

3 Englesbe MJ, Patel SP, He K, Lynch RJ, Schaubel DE, Harbaugh C, Holcombe SA, Wang SC, Segev DL and Sonnenday CJ: Sarcopenia and mortality after liver transplantation. J Am Coll Surg 211(2): 271-278, 2010. 
4 Peng PD, van Vledder MG, Tsai S, de Jong MC, Makary M, Ng J, Edil BH, Wolfgang CL, Schulick RD, Choti MA, Kamel I and Pawlik TM: Sarcopenia negatively impacts short-term outcomes in patients undergoing hepatic resection for colorectal liver metastasis. HPB 13(7): 439-446, 2011.

5 Lieffers JR, Bathe OF, Fassbender K, Winget $\mathrm{M}$ and Baracos VE: Sarcopenia is associated with postoperative infection and delayed recovery from colorectal cancer resection surgery. Br J Cancer 107(6): 931-936, 2012.

6 Peng P, Hyder O, Firoozmand A, Kneuertz P, Schulick RD, Huang D, Makary M, Hirose K, Edil B, Choti MA, Herman J, Cameron JL, Wolfgang $\mathrm{CL}$ and Pawlik TM: Impact of sarcopenia on outcomes following resection of pancreatic adenocarcinoma. J Gastrointest Surg 16(8): 1478-1486, 2012.

7 van Vledder MG, Levolger S, Ayez N, Verhoef C, Tran TC and Ijzermans $\mathrm{JN}$ : Body composition and outcome in patients undergoing resection of colorectal liver metastases. Br J Surg 99(4): 550-557, 2012.

8 Sabel MS, Lee J, Cai S, Englesbe MJ, Holcombe S and Wang S: Sarcopenia as a prognostic factor among patients with stage iii melanoma. Ann Surg Oncol 18(13): 3579-3585, 2011.

9 Montano-Loza AJ, Meza-Junco J, Prado CM, Lieffers JR, Baracos VE, Bain VG and Sawyer MB: Muscle wasting is associated with mortality in patients with cirrhosis. Clin Gastroenterol Hepatol 10(2): 166-173, 173.e1, 2012.

10 Tandon P, Ney M, Irwin I, Ma MM, Gramlich L, Bain VG, Esfandiari N, Baracos V, Montano-Loza AJ and Myers RP: Severe muscle depletion in patients on the liver transplant wait list: Its prevalence and independent prognostic value. Liver Transpl 18(10): 1209-1216, 2012.

11 Cruz-Jentoft AJ, Baeyens JP, Bauer JM, Boirie Y, Cederholm T, Landi F, Martin FC, Michel JP, Rolland Y, Schneider SM, Topinkova E, Vandewoude $\mathbf{M}$, Zamboni $\mathbf{M}$ and European Working Group on Sarcopenia in Older People: Sarcopenia: European consensus on definition and diagnosis: Report of the European Working Group on Sarcopenia in Older People. Age Ageing 39(4): 412-423, 2010.

12 Hasselager R and Gogenur I: Core muscle size assessed by perioperative abdominal ct scan is related to mortality, postoperative complications, and hospitalization after major abdominal surgery: A systematic review. Langenbecks Arch Surg 399(3): 287-295, 2014
13 Du Y, Karvellas CJ, Baracos V, Williams DC, Khadaroo RG, Acute $\mathrm{C}$ and Emergency Surgery G: Sarcopenia is a predictor of outcomes in very elderly patients undergoing emergency surgery. Surgery 156(3): 521-527, 2014.

14 Dirks RC, Edwards BL, Tong E, Schaheen B, Turrentine FE, Shada A and Smith PW: Sarcopenia in emergency abdominal surgery. J Surg Res 207: 13-21, 2017.

15 Levolger S, van Vugt JL, de Bruin RW and JN IJ: Systematic review of sarcopenia in patients operated on for gastrointestinal and hepatopancreatobiliary malignancies. Br J Surg 102(12): 1448-1458, 2015.

16 Matsushima K, Inaba K, Jhaveri V, Cheng V, Herr K, Siboni S, Strumwasser A and Demetriades D: Loss of muscle mass: A significant predictor of postoperative complications in acute diverticulitis. J Surg Res 211: 39-44, 2017.

17 Zuckerman J, Ades M, Mullie L, Trnkus A, Morin JF, Langlois Y, Ma F, Levental M, Morais JA and Afilalo J: Psoas muscle area and length of stay in older adults undergoing cardiac operations. Ann Thorac Surg 103(5): 1498-1504, 2017.

18 Yoshio M, Murakami G, Sato T, Sato S and Noriyasu S: The function of the psoas major muscle: Passive kinetics and morphological studies using donated cadavers. J Orthop Sci 7(2): 199-207, 2002

19 Miller AL, Min LC, Diehl KM, Cron DC, Chan CL, Sheetz KH, Terjimanian MN, Sullivan JA, Palazzolo WC, Wang SC, Hall KE and Englesbe MJ: Analytic morphomics corresponds to functional status in older patients. J Surg Res 192(1): 19-26, 2014.

20 Lidsky ME, Thacker JK, Lagoo-Deenadayalan SA and Scarborough JE: Advanced age is an independent predictor for increased morbidity and mortality after emergent surgery for diverticulitis. Surgery 152(3): 465-472, 2012. 